\title{
«Eine Übergangsregelung ist für die Einführung von SwissDRG zwingend»
}

Ab 2012 müssen alle Spitäler ihre Leistungen gemäss dem Fallpauschalensystem Swiss-
DRG abrechnen. Dies wurde im Rahmen der letzten Revision des KVG beschlossen. Aus Sicht der FMH gilt es, rasch eine national einheitliche Übergangsregelung für die Einführungsphase zu schaffen, damit sich Systemverzerrungen verhindern lassen. Ebenso fordert die FMH eine überzeugende Begleitforschung, um unerwünschte Effekte zu reduzieren und diese möglichst schnell zu korrigieren. Dr. med. PierreFrançois Cuénoud, Mitglied des FMH-Zentralvorstandes und Verantwortlicher Ressort SwissDRG, gibt Auskunft, was mit SwissDRG auf uns zukommt und was unbedingt noch zu tun ist.

Interview:

Jacqueline Wettstein

Korrespondenz: Jacqueline Wettstein $\mathrm{FMH}$

Abteilung Kommunikation Elfenstrasse 18

$\mathrm{CH}-3000$

Bern 13

Tel. 0313591111

Fax 0313591112

kommunikation@fmh.ch
Das Unbehagen von manchen Spitalärzten und medizinischen Fachpersonen scheint gross. Können Sie das nachvollziehen?

Pierre-François Cuénoud: Es ist verständlich, dass ein Systemwechsel Ängste und Befürchtungen auslösen kann. Gerade aber in der Schweiz haben Akutspitäler in 23 Kantonen bereits Erfahrungen mit einem Fallpauschalensystem gesammelt, diese werden auf nächstes Jahr quasi nur das Modell ändern. Wirklich neu ist das Fallpauschalensystem dann nur für Spitäler aus den Kantonen Baselland, Basel-Stadt und Solothurn. Und - die Autonomie der Medizin wird durch das Fallpauschalen-System nicht in Frage gestellt. Wie bisher entscheiden alleine die Fachleute, also auch Ärztinnen



Dr. med. Pierre-François Cuénoud ist Mitglied des FMH-Zentralvorstandes und der Verantwortliche für das Ressort SwissDRG. und Ärzte, über die Notwendigkeit medizinischer Eingriffe.

Gerade in der Pädiatrie befürchtet man erhebliche Nachteile durch SwissDRG. Ist dem so?

Die Behandlung von Kindern ist oft sehr zeit- und damit auch kostenintensiv. Diese können deshalb im Fallpauschalensystem nicht einfach als kleine erwachsene Patienten eingestuft werden. Und Kinderspitäler und -kliniken müssen auch unter SwissDRG kostendeckend arbeiten können. Aufgrund der Erfahrungen aus Deutschland haben wir mit den pädiatrischen Fachgesellschaften - der Schweizerischen Gesellschaft für Pädiatrie, der Schweizerischen Gesellschaft für Kinderchirurgie, der Schweizerischen Gesellschaft für Neuropädiatrie - frühzeitig darauf hingearbeitet, dass die Behandlung von Kindern möglichst adäquat in SwissDRG abgebildet sein wird, und zahlreiche Anträge zur Verbesserung eingereicht.

Einen separaten Weg gibt es auch für die Psychiatrie und die Rehabilitation: Hier werden gänzlich neue Modelle ausserhalb von SwissDRG entwickelt. Diese werden jedoch erst in einigen Jahren bereit sein. Bis zur definitiven Einführung wird wie bisher abgerechnet.

Immer wieder hört man, dass die Datenqualität der Spitäler noch zu verbessern ist für die Kostengewichte der Fallpauschalen ...

Während alle Spitäler die Leistungsdaten erheben, bilden erst 46 Netzwerkspitäler ihre Kostendaten ab. Dies soll spätestens bis zur SwissDRG-Version 3.0, sie ist für 2014 vorgesehen, wesentlich verbessert werden, damit die Kostengewichte anhand von Schweizer Daten berechnet werden können und nicht mehr deutsche Kostengewichte beigezogen werden müssen. 
Ist die FMH an der konkreten Ausgestaltung des Fallpauschalensystems mitbeteiligt?

Ja, und zwar von Beginn an. Zusammen mit den medizinischen Fachgesellschaften hat die FMH bisher insgesamt zirka 2000 Anträge eingereicht, um SwissDRG optimal auszugestalten. 90 Prozent dieser Anträge sind bereits umgesetzt. Dadurch sind mit Beginn des Jahres 2011 ca. 7500 Positionen in die Prozedurenklassifikation aufgenommen worden. Das alles war dank dem grossen Engagement der Fachgesellschaften und Dachverbände möglich, sowie dem SwissDRG-Team der FMH. Ihnen allen möchte ich herzlich danken! Damit zeigt sich auch deutlich, dass die Ärzteschaft eine hohe Glaubwürdigkeit geniesst. Wir werden auch die weitere Ausgestaltung von SwissDRG mitprägen, und zwar kooperativ, kompetent und sicherlich auch kritisch. Gerade auch letzteres ist unsere Aufgabe!

Wie steht es mit den Zusatzentgelten?

Wir setzen uns seit langem dafür ein, dass Zusatzentgelte geschaffen werden. Zusatzentgelte sind für die leistungsgerechte Vergütung von teuren Medikamenten, Blutprodukten, Implantaten und kostenintensiven Behandlungsmethoden unerlässlich. Sie tragen dazu bei, dass das DRG-System übersichtlich bleibt, und sie vermeiden unzählige neue Fallgruppen. Insgesamt machen sie lediglich zwei bis drei Prozent des gesamten Leistungsvolumens aus. Die gute Erfahrung aus Deutschland, dort gibt es 146 Zusatzentgelte, wollen wir auch im Schweizer Fallpauschalensystem abgebildet haben. Die Schweizer Einführungsversion der DRG sieht lediglich fünf Zusatzentgelte vor, was aus unserer Sicht klar ungenügend ist. Damit wird es auch zu Verzerrungen kommen. Noch ausstehend ist die Prüfung unserer Anträge $\mathrm{zu}$ den Zusatzentgelten durch die SwissDRG AG. Weitere Zusatzentgelte werden jedoch erst in die DRG-Version 2.0 einfliessen und damit im Jahr 2013 Realität sein.

Die FMH fordert eine national einheitliche Übergangsregelung ...

Ja genau, denn SwissDRG ist ein lernendes System und es gibt noch jede Menge offener Fragen. Diese werden nicht auf einen bestimmten Stichtag, etwa den

\section{SwissDRG - leistungsbezogene \\ Fallpauschalen}

Am 1. Januar 2012 tritt an Schweizer Spitälern ein neues Tarifsystem flächendeckend in Kraft: Ab dann werden stationäre Spitalleistungen mit dem Fallpauschalensystem SwissDRG (Swiss Diagnosis Related Groups) abgerechnet. Die Spitäler erhalten für jeden Spitalaufenthalt, d.h. für jeden Fall, eine pauschale Vergütung. Dazu wird jeder Fall aufgrund von bestimmten Kriterien - zum Beispiel aufgrund der Hauptdiagnose, der Nebendiagnosen, der Behandlung oder des Schweregrads - einer bestimmten Fallgruppe zugeordnet.
1. Januar 2012, gelöst sein. Es braucht deshalb zwingend eine national einheitliche Übergangsregelung während der Einführungsphase! Nur so können Systemverzerrungen abgefedert werden. Damit lässt sich verhindern, dass gut arbeitende Spitäler Verluste schreiben, nur weil ihr Behandlungsspektrum im DRG-System noch nicht genügend abgebildet ist. Spitäler benötigen deshalb finanzielle Garantien, damit sie weiterhin eine qualitativ hochstehende Versorgung bieten können. National einheitlich muss sie deshalb sein, weil individuelle Vereinbarungen zu einem Ungleichgewicht und damit zu Wettbewerbsverzerrungen führen.

Wer kommt für die ärztliche Weiterbildung unter SwissDRG auf?

Die Finanzierung der ärztlichen Weiterbildung ist aufgrund der föderalen Struktur des schweizerischen Gesundheitswesens höchst komplex. Bund und Kantone haben im Rahmen des Dialogs zur Nationalen Gesundheitspolitik (NGP) im vergangenen Jahr die Plattform «Zukunft ärztliche Bildung» ins Leben gerufen, die sich derzeit dem Themenschwerpunkt «Finanzierung der ärztlichen Weiterbildung» widmet. Neben der FMH bzw. dem SIWF sind alle wichtigen Stakeholder einbezogen, so u.a. das BAG, die GDK, die EDK, H+, der VSAO, der VLSS, santésuisse und die MEBEKO. Die Prüfung verschiedener Modelle ist im Gang. Im Vordergrund steht die Schaffung von zielgerichteten Anreizen, damit die Weiterbildungsstätten eine qualitativ hochstehende Weiterbildung anbieten. Es muss in jedem Fall verhindert werden, dass die Assistenzärztinnen und Assistenzärzte unter dem Druck von DRG keine Zeit mehr für ihre Weiterbildung haben. Die Hauptverantwortung für die Bereitstellung der entsprechenden Budgets liegt bei den Kantonen. Mit einer Revision der VKL (Verordnung über die Kostenermittlung und die Leistungserfassung durch Spitäler und Pflegeheime in der Krankenversicherung) könnte erreicht werden, dass nicht nur die Kantone, sondern auch die Versicherer zur Finanzierung der ärztlichen Weiterbildung beitragen.

Wie steht es um die Begleitforschung?

Eine qualitativ hochstehende Gesundheitsversorgung ist für uns von höchster Priorität. Das gilt auch unter dem neuen Fallpauschalensystem. Die FMH hat bereits frühzeitig eine Begleitforschung gefordert. Denn nur mit einer solchen lassen sich Fehlentwicklungen rechtzeitig erkennen und beheben. Die FMH hat 2009 ein konkretes Konzept für die Etablierung der Begleitforschung aus Anlass der Einführung von SwissDRG vorgelegt. 2010 folgte die Ausschreibung einer wissenschaftlichen Untersuchung, welche die für die Ärzteschaft wichtigsten Themen behandeln soll: Haben die Ärzte im Spital noch genügend Zeit für ihre Patienten? Nimmt der administrative Aufwand zu? Wie entwickeln sich die Arzt-PatientenBeziehung und die Zusammenarbeit mit den anderen 
Gesundheitsfachleuten? Mit ersten Resultaten ist Ende $2011 \mathrm{zu}$ rechnen.

Wie viel hat die FMH bisher für SwissDRG investiert? Die FMH ist seit $2008 \mathrm{im}$ Besitz von acht Swiss-DRG-Aktien zu je 1000 Schweizer Franken. Dies entspricht 8\% des gesamten Aktienkapitals. Zudem haben wir 2008 und 2009 je ein Darlehen von 250000 Franken gewährt, und im letzten Jahr ein solches von 83333 Franken. Diese drei Darlehen werden voraussichtlich zwischen 2012 und 2014 zurückbezahlt.

Das Spital, in dem Sie arbeiten, rechnet seit 13 Jahren mit einem Fallpauschalensystem ab. Welches sind Ihre Erfahrungen damit?

Die Fallpauschalen haben wie ein Katalysator gewirkt: Durch die unabdingbar gewordenen Rationalisierungsmassnahmen haben sie Leistungserbringer, politische Behörden und schliesslich auch Patienten zu einer globaleren statt wie bisher regionalen Denkweise gezwungen. Während früher mehrere Spitäler im Wallis gleichzeitig eine OP-Dienstbereitschaft hatten und die jeweiligen Teams auch unausgelastet waren - das ist unbefriedigend und kostet - ist die medizinische Versorgung nun überzeugender strukturiert. So etwa im Bereich der Geburtshilfe: Anstatt zahlreiche kleine Geburtsabteilungen haben sich an weniger Standorten eigentliche Kompetenzzentren gebildet. Diese verfügen über mehr Personal und damit auch über mehr Wissen und Erfahrung. Davon profitieren vor allem auch die Patientinnen. Sie nehmen auch gerne eine längere Anreise in Kauf, weil sie wissen, dass sie kompetent behandelt werden. Und genau das ist unsere Aufgabe: unsere Patienten bestmöglich zu versorgen.
Die Position der FMH - klare Forderungen Die Einführung von SwissDRG bringt tiefgreifende Änderungen für das schweizerische Gesundheitswesen mit sich. Die FMH stellt dabei grundlegende Forderungen an den neuen Tarif:

- Begleitforschung etablieren - noch vor der SwissDRG-Einführung

- Versorgungsqualität sicherstellen - SwissDRG darf zu keiner Verschlechterung führen

- Therapeutische Freiheit erhalten - unter Berücksichtigung der WZW-Kriterien (Wirksamkeit, Zweckmässigkeit, Wirtschaftlichkeit)

- Datenschutz gewährleisten - dazu gehört auch die Wahrung des Arztgeheimnisses

- Tatsächliche Spitalkosten abdecken - durch transparente Konzeption des Abgeltungssystems

- Innovationen abbilden - so rasch wie möglich, damit das SwissDRG-System stets aktuell bleibt

- Medizinische Kriterien respektieren - der Entscheid für eine ambulante oder stationäre Behandlung muss jeweils primär nach medizinischen Kriterien erfolgen

- Transparente Leistungserfassung bereitstellen - mit möglichst einfachen und effizienten Erfassungsverfahren

- Ärztliche Weiterbildung gewährleisten - dazu gehört auch die Sicherstellung der Finanzierung.

Das vollständige Positionspapier der FMH zu SwissDRG finden Sie unter www.fmh.ch $\rightarrow$ TARIFE $\rightarrow$ SwissDRG $\rightarrow$ Position der FMH. 